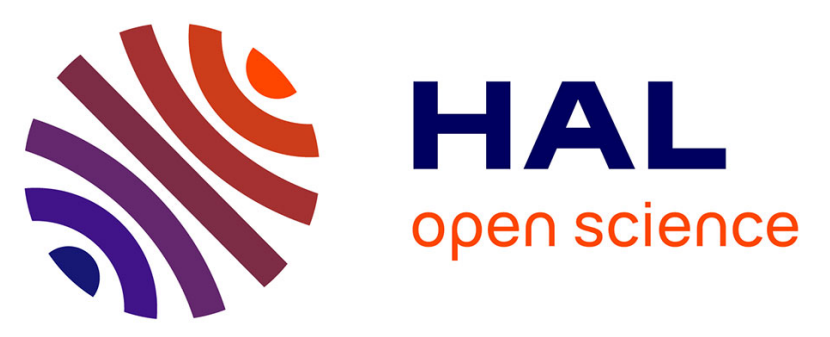

\title{
Importance of structural heart disease and diastolic dysfunction in heart failure with preserved ejection fraction assessed according to the ESC guidelines - A substudy in the Ka (Karolinska) Ren (Rennes) study
} Hans Persson, Erwan Donal, Lars H Lund, Dmitri Matan, Emmanuel Oger, Camilla Hage, Jean-Claude Daubert, Cecilia Linde

\section{To cite this version:}

Hans Persson, Erwan Donal, Lars H Lund, Dmitri Matan, Emmanuel Oger, et al.. Importance of structural heart disease and diastolic dysfunction in heart failure with preserved ejection fraction assessed according to the ESC guidelines - A substudy in the Ka (Karolinska) Ren (Rennes) study. International Journal of Cardiology, 2019, 274, pp.202-207. 10.1016/j.ijcard.2018.06.078 . hal-01935766

HAL Id: hal-01935766

https://hal-univ-rennes1.archives-ouvertes.fr/hal-01935766

Submitted on 19 Dec 2018

HAL is a multi-disciplinary open access archive for the deposit and dissemination of scientific research documents, whether they are published or not. The documents may come from teaching and research institutions in France or abroad, or from public or private research centers.
L'archive ouverte pluridisciplinaire HAL, est destinée au dépôt et à la diffusion de documents scientifiques de niveau recherche, publiés ou non, émanant des établissements d'enseignement et de recherche français ou étrangers, des laboratoires publics ou privés. 


\section{Importance of structural heart disease and diastolic dysfunction in heart} failure with preserved ejection fraction assessed according to the ESC Guidelines - a substudy in the Ka (Karolinska) Ren (Rennes) Study Hans Persson ${ }^{1}$, Erwan Donal ${ }^{2,4}$, Lars H. Lund ${ }^{3}$, Dmitri Matan ${ }^{1}$, Emmanuel Oger ${ }^{4}$, Camilla Hage $^{3}$, Jean-Claude Daubert ${ }^{2}$, Cecilia Linde ${ }^{3}$ on behalf of the KaRen Investigators.

${ }^{1}$ Karolinska Institutet, Department of Clinical Sciences, Danderyd Hospital, 18288 Stockholm, Sweden; ${ }^{2}$ University Hospital of Rennes, Departemente de Cardiologie \& CIC-IT U 804, Rennes, France; ${ }^{3}$ Karolinska Institutet, Karolinska University Hospital, Heart and Vascular Theme, 17176 Stockholm, Sweden; ${ }^{4}$ Clinical Investigation Centre of Rennes, INSERM CIC-0203, CHU de Rennes, Rennes, France 


\begin{abstract}
Aims: To study prevalence and prognostic importance of diagnostic echocardiographic variables in patients with suspected heart failure with preserved ejection fraction (HFpEF) in the prospective KaRen register study.
\end{abstract}

Methods and results: KaRen patients were included following an acute HF-presentation, using Framingham criteria, B-type natriuretic peptide (BNP) $>100 \mathrm{ng} / \mathrm{L}$ or N-terminal proBNP (NT-pro-BNP) $>300 \mathrm{ng} / \mathrm{L}$, and left ventricular (LV) ejection fraction $\geq 45 \%$.

Echocardiography was performed after 4-8 weeks and analysed at a core laboratory. In this substudy HFpEF was diagnosed according to the ESC guidelines for heart failure 2016.

A total of 539 patients were included with a follow-up after 4-8 weeks in 438 patients. Complete echocardiography and ECG were available in 356 patients. At least two abnormal echocardiographic criteria for HFpEF were found in 94\% (n=333). Echocardiographic signs of structural heart disease and diastolic dysfunction according to 4 criteria by ESC were found in $76 \%(n=270)$. Diastolic dysfunction was graded as mild in $30 \%(n=107)$, moderate in $27 \%$ $(n=97)$ or severe in $35 \%(n=124)$. After multivariate analyses with adjustment for age, gender, EF and natriuretic peptides we found two independent predictors of worse prognosis: presence of moderate and severe diastolic dysfunction (HR 1.8, CI 1.2-2.7, p=0.0037) and presence of a high number $(\geq 4)$ of abnormal diastolic parameters (HR 2.0, CI 1.3-3.1, $\mathrm{p}=0.0033)$.

Conclusion: The majority of KaRen patients with suspected HFpEF had diagnostic echocardiographic criteria for HFpEF according to ESC Guidelines. Our findings support using 2016 ESC HF guidelines for risk prediction in HFpEF. 
Key words: Heart failure with preserved ejection fraction, echocardiography, prognosis, diagnosis, diastolic dysfunction.

\section{Introduction}

Heart failure (HF) is an increasing health problem in the world, especially in the developed countries (1). HF is one of the most frequent causes of hospital admission $(2,3)$, and is also associated with high mortality (4). About 40-50\% of the patients with HF have preserved left ventricular (LV) ejection fraction (EF), named HFpEF, and this percentage is growing (5). Both morbidity and mortality in HFpEF are similar to those in HF with reduced EF (HFrEF), and a recent study from the KaRen group showed that non-cardiovascular co-morbidities have a great impact on prognosis in HFpEF (6). There is no established treatment for HFpEF (7). In 2007 the European Society of Cardiology (ESC) adopted a consensus statement (8) that recommended that the diagnosis of HFpEF should be based on a combination of biochemical (values of natriuretic peptides) and echocardiographical (such as mitral inflow and tissue Doppler variables, volume of the left atrium or mass of the left ventricle) parameters.

\section{Methods}

\section{Study design}

The Karolinska-Rennes (KaRen) study has been previously described (9). It is a prospective multicentre observational cohort study that aims to characterize clinical and echocardiographic characteristics in HFpEF and their prognostic importance (10). Patients were included in the KaRen study between 1 May 2007 and 1 December 2011; the inclusion took place in 10 French and 3 Swedish University hospitals. In short patients were included 
following an acute presentation of HF according to all of the following: the Framingham criteria (11), left ventricular ejection fraction (LVEF) $\geq 45 \%$ and elevated levels of natriuretic peptides: B-type natriuretic peptide (BNP) >100 ng/L or N-terminal pro-BNP (NT-pro-BNP) $\geq 300 \mathrm{ng} / \mathrm{L}$ within 72 hours of presentation. The study was approved by local ethic committees.

\section{Study purpose}

Our aim in this substudy was to investigate to what extent patients with clinical signs of HF and preserved EF fulfil the diagnostic criteria of HFpEF set by most recent ESC HF guidelines (12). These selected patients with assumed HFpEF underwent an extensive Doppler echocardiographic investigation in stable phase 4-8 weeks after admission to assess if the initial HFpEF diagnosis could be verified. Additionally, severity of diastolic dysfunction was evaluated. Finally, our aim was to investigate if these diagnostic parameters had an influence on the primary outcome of the KaRen study - mortality and hospitalizations for HF.

\section{Patients}

A total of 539 consecutive patients were enrolled in the KaRen study. Of these, 438 patients had a follow-up visit after 4-8 weeks. An electrocardiogram (ECG) was available in 393 patients and a complete echocardiography in 356 patients of them (9), so there were totally 413 patients with either analysable ECG or echocardiogram. Our analysis is restricted to the 356 patients with analysable echocardiogram.

\section{Follow up}

The quantitative analysis was performed in a 'Core laboratory' (CIC-IT 1414, CHU Rennes, France) (13). A 12-lead ECG was also performed at the follow-up visit and analyzed by 
another core laboratory (Karolinska University Hospital, Stockholm, Sweden). The primary study endpoint was time to death from all causes or first hospitalisation for HF.

\section{Echocardiography}

The examinations were performed according to a checklist on the same type of machine (ViVid 7, manufactured by GE Healthcare, Horten, Norway). In this paper our findings were put in relation to the eight criteria defined by the ESC HF guidelines (12). Cardiac structure was characterized by left ventricular mass index (LVMI) and left atrial volume index (LAVI). The diastolic LV function was characterized by isovolumetric relaxation time (IVRT), deceleration time (DT), ratio of mitral E to A velocity (E/A), average mitral tissue Doppler e' velocity, ratio of mitral Doppler E velocity to average mitral tissue Doppler e'-velocity (E/e') and peak flow velocity of tricuspid regurgitation (TR). Systolic LV function was characterized by LVEF. Right-sided cardiac catheterisation was not part of the protocol.

\section{Diagnosis of HFPEF in a model according to present ESC guidelines}

The ESC HF Guidelines list both clinical and the aforementioned echocardiographic criteria for diagnosing HFpEF (12). First, the patient should have symptoms and signs typical of HF. Second, echocardiography should show normal or only slightly reduced LVEF (reduced

LVEF is defined as $<50 \%$ ). Finally, structural heart disease, such as LV hypertrophy and enlargement of the left atrium (LA) or direct and indirect measures of diastolic LV dysfunction, such as elevated E/e' or low e' or tricuspid regurgitation velocity should be used (9).

Previous definitions of HFpEF Consensus statement by ESC 
According to a more detailed earlier ESC Consensus statement (8) patients should have symptoms or signs of HF, normal or mildly reduced left ventricular systolic function (normal LVEF defined as $>50 \%$ ) and non-enlarged LV (LVDVI $\left.<97 \mathrm{~mL} / \mathrm{m}^{2}\right)$. Then there are several pathways leading to the HFpEF diagnosis. They include elevated filling pressures, such as measured invasively by mean pulmonary capillary wedge pressure $>12 \mathrm{mmHg}$ or signs of abnormal LV relaxation, filling, or diastolic stiffness. Secondly, the diagnosis can be based on echocardiography showing increased E/e' ratio (>15). If E/e' is only moderately increased, the diagnosis should be made with either measuring of biomarkers (NT-pro-BNP > $220 \mathrm{ng} / \mathrm{L}$ or $\mathrm{BNP}>200 \mathrm{ng} / \mathrm{L}$ is sufficient for diagnosing $\mathrm{HFpEF}$ ) or other echocardiographic investigations regarding LV mass, LA volume or diastolic LV dysfunction.

\section{Additional Echo guidelines (ASE and EACVI)}

Echocardiographic guidelines for evaluation of left ventricular diastolic function are recently presented from the American Society of Echocardiography (ASE) and the European Association of Cardiovascular Imaging (EACV) (14). They recommend evaluation of following variables when assessing the diastolic function of the LV: e', average E/e', LAVI and peak flow velocity of tricuspid regurgitation (TR). These guidelines advocate the use of numerous variables to add diagnostic value.

\section{Definitions and cut-offs for the present analysis}

Based on the aforementioned criteria in the ESC Guidelines, the Consensus statement and the ASE/EACV Guidelines we have chosen the echocardiographic cut-off 
values for this KaRen substudy. The cut-off values for the eight echocardiographic parameters are defined in Appendix 1. For HF biomarkers we used following cut-off values in the KaRen study: BNP >100 ng/L and NT-pro-BNP>300 ng/L.

\section{Grading of diastolic function}

The classification of diastolic function was based on the ESC algorithm and its cut-offs (8, 12) and also on a semi-quantitative grading of diastolic function $(9,14)$. A similar classification method has previously been used by the Mayo Clinic group (15). Classification was as follows: 0) normal, 1) relaxation abnormality (mild dysfunction), 2) pseudonormalisation (moderate dysfunction), and 3) restrictive filling abnormality (severe dysfunction). A relaxation abnormality was based on presence of at least one abnormal mitral inflow parameter $(\mathrm{E} / \mathrm{A}<0.5$, IVRT $>110 \mathrm{~ms}$ or $\mathrm{DT}>280 \mathrm{~ms})$ or dilatation of the left atrium (increased LAVI).

To distinguish pseudo-normal from normal diastolic function, E/e' and TR were used and, for the diastolic function to be classified as pseudo-normal, one of these parameters had to be elevated ( $\geq 13$ or $\geq 2.8$ respectively). Besides that, at least one of the following three mitral inflow parameters had to be within the normal range: E/A (0.5-2), IVRT (55-110 ms) or DT (150-280ms). For diagnosis of the restrictive pattern, the cut-offs for E/e' and TR were the same as for pseudo-normalisation, but a value for at least one of the mitral inflow parameters had to be pathological: E/A $>2$, IVRT $<55 \mathrm{~ms}$ or DT $<150 \mathrm{~ms}$. Our diagnostic criteria are summarized in Appendix 2. The patients not fulfilling the abovementioned diagnostic criteria were assessed to have normal diastolic LV function.

In patients with atrial fibrillation where E/A could not be measured, the diastolic function was assessed using following parameters: LAVI, DT, IVRT and E/e' according to the 
abovementioned algorithm. This is the method recommended by the current EACVI/EHRA Consensus Document (16).

\section{Statistical analysis}

The categorical variables were expressed as $\mathrm{n}(\%)$ and the continuous variables were expressed as mean \pm standard deviation. The data were split according to the definitions of cut-offs above. For prognostic assessment of the outcome predictors, univariate and multivariate Cox regressions were used. The multivariate analysis was performed to assess if there was additive prognostic value of adding echo variables and diastolic grading or structural disease classifications adjusted for age, gender, ejection fraction and level of natriuretic peptides.

The data sets were analysed using the standard SAS procedures (PHREG Procedure and LIFETEST Procedure). Considering the limited available number of patients and that some missing data were found in the echo parameters, we did multiple imputations (PROC MI) using a fully conditional specification method that performed a regression method to impute missing values for continuous variables. After 25 complete data sets were analyzed using standard SAS procedures (PROC PHREG), we used the MIANALYZE procedure that combined the results of theses 25 analyses and generates valid statistical inferences. Hazard ratios $(95 \% \mathrm{CI})$ estimated through Cox regression (PROC PHREG) were used as measure of association with the primary study endpoint.

\section{Ethics}


This study is performed in accordance to the Declaration of Helsinki and is approved by French and Swedish ethics committees and by the CNIL (Comité National Informatique et Libertés) in France.

\section{Results}

\section{Patients'characteristics}

Demographic and clinical characterics of the KaRen study population has been published (9, 10) - they are summarized in Appendix 3. The patients were elderly and many of them had a previous history of cardiac or other diseases. A clear majority of the patients had symptoms of heart failure.

The patients were followed up by telephone calls and reviews of chart or death registry every 6 month for 18 months after closure of enrolment, so each patient had a follow-up time of at least 18 months. With a mean follow-up of 28 months 156 patients $(43.8 \%)$ reached the combined primary endpoint (10). Thirty-nine per cent of the patients $(n=171)$ were diagnosed with atrial arrhythmia after the examination of their ECGs (9), mainly atrial fibrillation.

In Table 1 the baseline clinical characteristics are presented, both overall and stratified by number of echo abnormalities.

\section{Echocardiography after 4-8 weeks}

Prevalence of abnormal echocardiographic variables was high, as summarized in Appendix 3. For most of the included patients the echocardiographic parameters were pathological. Signs of structural dysfunction (LAVI $\geq 34 \mathrm{ml} / \mathrm{m}^{2}$ or $\mathrm{LVMI} \geq 95 / 115 \mathrm{~g} / \mathrm{m}^{2}$ ) were found in $92 \%$ ( $n=328$ ). Signs of diastolic dysfunction $\left(E / e^{\prime} \geq 13\right.$, or e' $<9 \mathrm{~cm} / \mathrm{s}$, or $T R \geq 2.8 \mathrm{~m} / \mathrm{s}$ ) were found 
in $82 \%(n=290)$. Signs of both structural and diastolic dysfunction were found in $76 \%$ $(n=270)$.

In Table 2 the key echo variables are presented.

At least one abnormal echocardiographic criterion for HFpEF was found in $98 \%(n=351)$ and 94\% $(n=333)$ had at least two pathological criteria. One to three abnormal criteria were found in $38 \%(n=134)$, four criteria in $24 \%(n=84), 5-8$ abnormal criteria in $39 \%(n=138)$ of the patients.

According to the above-mentioned classification method, grading of the diastolic function was performed by D. Matan and H. Persson. Out of the total of 356 patients examined with echocardiography, 107 patients (30\%) had mild diastolic dysfunction (relaxation abnormality), 97 patients (27\%) had moderate diastolic dysfunction (pseudo-normalisation) and 124 patients (35\%) had severe diastolic dysfunction (restrictive pattern). Twenty-four patients $(7 \%)$ were assessed to have normal diastolic LV function. Four patients (1\%) were assessed as non-classifiable due to diverging/conflicting data; they were excluded from further analysis. Thus, 352 (99\%) of the 356 patients were possible to classify based on available parameters.

\section{Influence of parameters on outcome}

After univariate analysis, it was shown that abnormal values of the following diastolic parameters have significant $(\mathrm{p}<0.05)$ impact on the outcome: E/A, E/e', TR and LVMI. The outcome was also dependent on the number of abnormal diastolic parameters as defined by separation of number of abnormal variables into tertiles (with significant impact when four or more parameters were pathological). Thirdly, diastolic dysfunction showed a graded 
association to outcome, where patients with severe diastolic dysfunction had significantly worse outcome compared to those with normal diastolic function.

A multivariable analysis with adjustment for age, gender and EF was performed showing that the outcome was significantly worse for the patients with moderate and severe diastolic dysfunction compared to normal and mild dysfunction. The outcome was also significantly worse for the patients with echocardiographic signs of both structural and diastolic dysfunction compared to those without such signs. Finally, the outcome was significantly worse when the number of the pathological diastolic parameters was four or more. The results of the multivariate analysis are summarized in Table 3 and in Figure 1. These results remained unchanged for number of abnormal variables and grading of diastolic after further adjustment for BNP/ NT-pro-BNP values.

\section{Discussion}

\section{Short summary of study and results}

Our findings suggest that clinical diagnosis of HF based on Framingham criteria with the addition of a modest increase in natriuretic peptides can be used for finding a cohort of patients with objective echocardiographic and diagnostic criteria for $\mathrm{HFpEF}$ according to the most recent guidelines from the ESC and verified by extensive echocardiography in a core lab centre. Our prognostic results strengthen the use of parameters and cut-offs recommended by ESC, as we could show that both the number of pathological diastolic parameters, and moderate and severe diastolic dysfunction are independent predictors of prognosis, respectively. Our finding support previous studies, such as I-PRESERVE (17), TOPCAT (18) and CHARM (18), clinical trials that showed the prognostic importance of echocardiographic signs of HFpEF. 


\section{Patients}

Out of totally 539 patients included in the KaRen study, 183 were not examined with echocardiography and ECG and thus excluded from this substudy. However, as the vast majority of the patients in the substudy had pathological echocardiographic criteria, there is a high probability that also the patients lost to follow-up would be diagnosed with HFpEF if a full diagnostic echocardiographic study had been performed. Apart from higher mean age (80 \pm 9 vs $76 \pm 9$ years; $p=0.006$ ), there were no statistically significant differences between the patients who returned for the follow-up visit and those who did not (9). 


\section{Echocardiography and diagnostic algorithms for HFpEF}

The inclusion criteria in the KaRen study - to use both clinical and echocardiographic data together with natriuretic peptides - are in accordance with the recommendations in the ESC Guidelines (12). The echocardiographic cut-off values we used are consistent with those suggested in the Guidelines and in previous studies $(14,19)$. Some of the parameters, particularly for diastolic function, are relatively difficult to achieve in every patient, which makes diagnostic algorithms of several alternative parameters or biomarker data attractive for clinical use (14). As can be seen in the present study, using 8 variables as suggested by ESC makes a diagnosis possible in most HFpEF patients with a preliminary diagnosis based only on clinical data, preserved $\mathrm{EF}$ and a modest increase in natriuretic peptide level. By using several diagnostic parameters we were able to classify most of the patients, a similar finding compared to a previous study performed on a similar clinical population with echoes interpreted by a core lab (19). If we only used clinical diagnosis of $\mathrm{HF}$ and preserved EF, we would have included a large proportion of patients with normal LV function and a subsequent very low mortality and low risk of future heart failure (19-21). Further, the prognostic value of most of these parameters strengthens the case for using them. However, in a recent paper from the KaRen study only E/e' showed an independent prognostic value (10) when creating a full predictive model including all available variables in the study. However, the present analysis is more adapted to what is generally available in the clinical situation.

Many patients in KaRen showed an enlarged LV, and also other measures of depressed LV longitudinal function (9). Therefore, by only including preserved EF in the primary diagnosis a relatively large population of patients with enlarged LV volume would be diagnosed as HFpEF, which is not in accordance with the ESC guidelines. 
The patients with suspected HFpEF can be selected by clinical signs and the levels of natriuretic peptides, but echocardiography is necessary to confirm the diagnosis by verifying cardiac dysfunction, in this case with evidence of LV dysfunction. Several recent studies have confirmed the necessity of echocardiography in diagnosing HFpEF (22-24). However, many clinicians still diagnose HF clinically without echocardiography, and using natriuretic peptides for screening is thus important (25). Echocardiographic access is a strong limitation due to poor availability in most countries. Selection of patients to echocardiography by using increase in natriuretic peptides is important, because of their strong negative predictive value when under ESCs threshold levels.

We also examined whether only LAVI by itself could be used for prognosing of the outcome in HFpEF, and concluded that it was not the case, although LAVI was related to diastolic dysfunction (Appendix 4). This is consistent with the findings in the CHARMES study.

\section{Natriuretic peptides}

Recent studies have shown that analysis of biomarkers, both BNP and NT-pro-BNP, can be used for diagnosing of $\operatorname{HFpEF}(19,20,26,27)$, often in conjunction with use of conventional echocardiographic parameters as suggested in the ESC Guidelines and in the Consensus Statement. Further, both these biomarkers can be used as predictors of prognosis in HFpEF, as elevated values in BNP and NT-pro-BNP are associated with increased frequency of adverse cardiovascular events $(28,29)$. In the I-PRESERVE study NT-pro-BNP was the strongest outcome predictor for all cardiovascular events (30). In the present study we show additive prognostic role of using echocardiography on top of using natriuretic peptides.

\section{Clinical diagnosis and Framingham criteria}


In the KaRen study the presence of clinical signs of HF according to the Framingham criteria was mandatory for inclusion. Clinical signs of HF are still important for correct diagnosis and prognosis in heart failure $(31,32)$, as has been shown in numerous studies from 1970s and onwards $(26,33)$. Whether a less formal clinical diagnosis than the Framingham criteria are useful to the same degree is not certain, but a recent HF hospitalization and time from this event was a very strong predictor for future events in patients with chronic heart failure with reduced and preserved EF (34).

\section{Outcome}

After multivariate analyses with adjustment for age, gender and EF and natriuretic peptides we found two factors that were independent predictors of worse prognosis: presence of moderate and severe diastolic dysfunction and presence of a high number (four or more) of pathological diastolic parameters. Presence of structural heart disease and presence of diastolic dysfunction using only 4 criteria (LAVI, LVMI, E/e' and TR) did not show independent prognostic value when adjusted for natriuretic peptides. Further studies are required for better understanding of the predictive value of different echocardiographic parameters in HFpEF.

\section{Study limitations}

Several limitations can be identified in this study. First, there is no comparator, non-HFpEF, group in the KaRen study, which may diminish the strength of showing prognostic utility for the diagnostic strategy. However, in our opinion, the KaRen study uses real world data interpreted in accordance with the ESC diagnostic algorithm, which provides an insight in diagnosis and prognosis of HFpEF, which adds to insights from clinical trials with more selected HFpEF patients (16-18). 
Secondly, in our study we do not account for age-related variation in the parameters of diastolic dysfunction, although such variation has been previously described (35). However, the results from the CHARM echocardiographic study carried out by this group (19) have shown that using age-adjusted and non age-adjusted normal values of diastolic dysfunction does not make a significant difference to prognostic utility and proportion of patients in the respective subgroups of diastolic dysfunction in the same HFpEF population (36).

Thirdly, KaRen patients were included based on EF $\geq 45 \%$ and not $>50 \%$ as is now the present recommendation by ESC. However, we kept the few $(n=9)$ patients with $\mathrm{EF} \leq 50 \%$ in the present analysis because they belong to the new category introduced by ESC with heart failure and midrange ejection fraction (HFmrEF). According to the ESC Guidelines the same objective diagnostic criteria should be used for HFmrEF as the ones used in this analysis.

Fourthly, this study does not include an age-matched control group without heart failure diagnosis, which is a weakness as to possibility to compare the echocardiographic findings in HFpEF population and in healthy individuals. However, our findings can be compared to the normal reference ranges of echocardiographic parameters that have been gathered in the NORRE study (37), the largest European registry study carried out by the the European Association of Cardiovascular Imaging (EACVI).

Finally, it should be noticed that the generalizability of the study findings is limited to $\mathrm{HFpEF}$, as one of the inclusion criteria of the study was $\mathrm{EF} \geq 45 \%$.

\section{Conclusions}

In summary, we conclude that almost all patients with suspected HF based on Framingham criteria, modest increase of natriuretic peptides and $\mathrm{EF} \geq 45 \%$ met objective diagnostic 
echocardiographic criteria for HFpEF as defined by the ESC guidelines. Our findings support the use of the ESC guidelines for risk prediction in HFpEF.

Funding: The KaRen study is an academic study financed by the Karolinska Institute,

Sweden, and the Rennes University, France. The study was partly supported by grants from

Fédération française de cardiologie/Société française de cardiologie, France, AIG Europe

Limited, Stockholm, Sweden and Medtronic Bakken Research Center, Maastricht, The

Netherlands. The KaRen study also received a grant from Medtronic Europe.

Acknowledgements: Contribution from D. Matan to this substudy within the KaRen study: hypothesis and aim for the present analysis, drafting and revisions of the manuscript together with $\mathrm{H}$. Persson, classification of diastolic dysfunction, statistical considerations, reviewing current guidelines for HFpEF diagnosis.

Conflict of interest: The authors report no relationships that could be construed as a conflict of interest.

* The list of the KaRen investigators is provided in Appendix 5. 


\section{References:}

1. Mozaffarian D, Benjamin EJ, Go AS, et al. Heart disease and stroke statistics-2015 update: a report from the american heart association. Circulation. 2015;131(4):e29-e322.

2. Giamouzis G, Kalogeropoulos A, Georgiopoulou V, et al. Hospitalization epidemic in patients with heart failure: risk factors, risk prediction, knowledge gaps, and future directions. Journal of cardiac failure. 2011;17(1):54-75.

3. Dunlay SM, Redfield MM, Weston SA, et al. Hospitalizations after heart failure diagnosis a community perspective. Journal of the American College of Cardiology. 2009;54(18):1695702.

4. Bytyci I, Bajraktari G. Mortality in heart failure patients. Anadolu kardiyoloji dergisi : $A K D=$ the Anatolian journal of cardiology. 2015;15(1):63-8.

5. Heidenreich PA, Albert NM, Allen LA, et al. Forecasting the impact of heart failure in the United States: a policy statement from the American Heart Association. Circulation Heart failure. 2013;6(3):606-19.

6. Lund LH, Donal E, Oger E, et al. Association between cardiovascular vs. non-cardiovascular comorbidities and outcomes in heart failure with preserved ejection fraction. European journal of heart failure. 2014;16(9):992-1001.

7. Borlaug BA, Paulus WJ. Heart failure with preserved ejection fraction: pathophysiology, diagnosis, and treatment. European heart journal. 2011;32(6):670-9.

8. Paulus WJ, Tschope C, Sanderson JE, et al. How to diagnose diastolic heart failure: a consensus statement on the diagnosis of heart failure with normal left ventricular ejection fraction by the Heart Failure and Echocardiography Associations of the European Society of Cardiology. European heart journal. 2007;28(20):2539-50.

9. Donal E, Lund LH, Oger E, et al. Baseline characteristics of patients with heart failure and preserved ejection fraction included in the Karolinska Rennes (KaRen) study. Archives of cardiovascular diseases. 2014;107(2):112-21.

10. Donal E, Lund LH, Oger E, et al. New echocardiographic predictors of clinical outcome in patients presenting with heart failure and a preserved left ventricular ejection fraction: a subanalysis of the Ka (Karolinska) Ren (Rennes) Study. European journal of heart failure. 2015;17(7):6808.

11. McKee PA, Castelli WP, McNamara PM, Kannel WB. The natural history of congestive heart failure: the Framingham study. The New England journal of medicine. 1971;285(26):1441-6. 
12. Ponikowski P, Voors AA, Anker SD, et al. 2016 ESC Guidelines for the diagnosis and treatment of acute and chronic heart failure: The Task Force for the diagnosis and treatment of acute and chronic heart failure of the European Society of Cardiology (ESC). Developed with the special contribution of the Heart Failure Association (HFA) of the ESC. European journal of heart failure. 2016;18(8):891-975.

13. Donal E, Lund LH, Linde C, et al. Rationale and design of the Karolinska-Rennes (KaRen) prospective study of dyssynchrony in heart failure with preserved ejection fraction. European journal of heart failure. 2009;11(2):198-204.

14. Nagueh SF, Smiseth OA, Appleton CP, et al. Recommendations for the Evaluation of Left Ventricular Diastolic Function by Echocardiography: An Update from the American Society of Echocardiography and the European Association of Cardiovascular Imaging. European heart journal cardiovascular Imaging. 2016.

15. Bursi F, Weston SA, Redfield MM, et al. Systolic and diastolic heart failure in the community. Jama. 2006;296(18):2209-16.

16. Donal E, Lip GY, Galderisi M, et al. EACVI/EHRA Expert Consensus Document on the role of multimodality imaging for the evaluation of patients with atrial fibrillation. European heart journal cardiovascular Imaging. 2016;17(4):355-83.

17. Zile MR, Gottdiener JS, Hetzel SJ, et al. Prevalence and significance of alterations in cardiac structure and function in patients with heart failure and a preserved ejection fraction. Circulation. 2011;124(23):2491-501.

18. Shah AM, Claggett B, Sweitzer NK, et al. Prognostic Importance of Changes in Cardiac Structure and Function in Heart Failure With Preserved Ejection Fraction and the Impact of Spironolactone. Circulation Heart failure. 2015;8(6):1052-8.

19. Persson $\mathrm{H}$, Lonn $\mathrm{E}$, Edner $\mathrm{M}$, et al. Diastolic dysfunction in heart failure with preserved systolic function: need for objective evidence:results from the CHARM Echocardiographic SubstudyCHARMES. Journal of the American College of Cardiology. 2007;49(6):687-94.

20. Kasner M, Gaub R, Westermann D, et al. Simultaneous estimation of NT-proBNP on top to mitral flow Doppler echocardiography as an accurate strategy to diagnose diastolic dysfunction in HFNEF. International journal of cardiology. 2011;149(1):23-9.

21. Wachter R, Edelmann F. Diagnosis of heart failure with preserved ejection fraction. Heart failure clinics. 2014;10(3):399-406.

22. Dokainish $\mathrm{H}$. Left ventricular diastolic function and dysfunction: Central role of echocardiography. Global cardiology science \& practice. 2015;2015:3.

23. Kovacs SJ. Diastolic function in heart failure. Clinical Medicine Insights Cardiology. 2015;9(Suppl 1):49-55. 
24. Pellicori P, Cleland JG. Update on management of heart failure with preserved ejection fraction. Current opinion in cardiology. 2015.

25. Kelder JC, Cowie MR, McDonagh TA, et al. Quantifying the added value of BNP in suspected heart failure in general practice: an individual patient data meta-analysis. Heart. 2011;97(12):95963.

26. Paul B, Soon KH, Dunne J, De Pasquale CG. Diagnostic and prognostic significance of plasma Nterminal-pro-brain natriuretic peptide in decompensated heart failure with preserved ejection fraction. Heart, lung \& circulation. 2008;17(6):497-501.

27. Grewal J, McKelvie R, Lonn E, et al. BNP and NT-proBNP predict echocardiographic severity of diastolic dysfunction. European journal of heart failure. 2008;10(3):252-9.

28. Grewal J, McKelvie RS, Persson $\mathrm{H}$, et al. Usefulness of N-terminal pro-brain natriuretic Peptide and brain natriuretic peptide to predict cardiovascular outcomes in patients with heart failure and preserved left ventricular ejection fraction. The American journal of cardiology. 2008;102(6):733-7.

29. Jhund PS, Anand IS, Komajda M, et al. Changes in N-terminal pro-B-type natriuretic peptide levels and outcomes in heart failure with preserved ejection fraction: an analysis of the I-Preserve study. European journal of heart failure. 2015.

30. McKelvie RS, Komajda M, McMurray J, et al. Baseline plasma NT-proBNP and clinical characteristics: results from the irbesartan in heart failure with preserved ejection fraction trial. Journal of cardiac failure. 2010;16(2):128-34.

31. Desta L, Jernberg T, Lofman I, et al. Incidence, temporal trends, and prognostic impact of heart failure complicating acute myocardial infarction. The SWEDEHEART Registry (Swedish WebSystem for Enhancement and Development of Evidence-Based Care in Heart Disease Evaluated According to Recommended Therapies): a study of 199,851 patients admitted with index acute myocardial infarctions, 1996 to 2008. JACC Heart failure. 2015;3(3):234-42.

32. Lala A, Hochman JS. Standing the test of time. JACC Heart failure. 2015;3(3):243-4.

33. Vaur L, Gueret $P$, Lievre M, Chabaud S, Passa P, study DSG. Development of congestive heart failure in type 2 diabetic patients with microalbuminuria or proteinuria: observations from the DIABHYCAR (type 2 DIABetes, Hypertension, CArdiovascular Events and Ramipril) study. Diabetes care. 2003;26(3):855-60.

34. Bello NA, Claggett B, Desai AS, et al. Influence of previous heart failure hospitalization on cardiovascular events in patients with reduced and preserved ejection fraction. Circulation Heart failure. 2014;7(4):590-5.

35. Marwick TH, Gillebert TC, Aurigemma G, et al. Recommendations on the use of echocardiography in adult hypertension: a report from the European Association of Cardiovascular Imaging 
(EACVI) and the American Society of Echocardiography (ASE)dagger. European heart journal cardiovascular Imaging. 2015;16(6):577-605.

36. Kolias TJ. Diastolic dysfunction and heart failure. Journal of the American College of Cardiology. 2007;50(1):79; author reply -80.

37. Lancellotti P, Badano LP, Lang RM, et al. Normal Reference Ranges for Echocardiography: rationale, study design, and methodology (NORRE Study). European heart journal cardiovascular Imaging. 2013;14(4):303-8. 


\section{Figure legends}

Figure 1. Probability of adverse event according to number of abnormal diastolic parameters (Kaplan-Meier Curve with univariate analysis). Tertile 1 (0-3 abnormal variables) versus Tertile 2 and 3 (4-8 abnormal variables).

Abbreviations: $\mathrm{HF}=$ heart failure, $\mathrm{HR}=$ hazard ratio, $\mathrm{CI}=$ confidence interval. 
Table 1. Baseline clinical characteristics, both overall and stratified by number of echo abnormalities

\begin{tabular}{|c|c|c|c|c|c|}
\hline \multirow[t]{3}{*}{ Characteristic } & \multirow{3}{*}{$\begin{array}{l}\text { Missing } \\
\mathrm{N}\end{array}$} & \multirow{3}{*}{$\begin{array}{l}\text { Overall } \\
\mathrm{N}=356\end{array}$} & \multicolumn{3}{|c|}{ Number of echo abnormalities } \\
\hline & & & $<4$ & 4 & 5 to 8 \\
\hline & & & $N=106$ & $\mathrm{~N}=94$ & $N=156$ \\
\hline Age, years & 0 & $76(9)$ & $73(10)$ & $76(9)$ & $78(8)$ \\
\hline Gender, female & 0 & $201(56.5)$ & $54(50.9)$ & $53(56.4)$ & $94(60.3)$ \\
\hline BMI, $\mathrm{kg} / \mathrm{m}^{2}$ & 17 & $29.5(6.5)$ & $30.7(7.2)$ & $29.9(6.6)$ & $28.5(5.8)$ \\
\hline AF or flutter & 34 & $118(36.6)$ & $31(34.8)$ & $35(40.7)$ & $52(35.4)$ \\
\hline Hypertension & 1 & $282(79.4)$ & $73(69.9)$ & $82(88.2)$ & $127(81.4)$ \\
\hline Prior HF & 4 & $136(38.6)$ & $28(26.4)$ & $43(46.2)$ & $65(42.5)$ \\
\hline CAD & 9 & $118(34.0)$ & 37 (35.6) & $36(39.1)$ & $45(29.8)$ \\
\hline Diabetes & 0 & $115(32.3)$ & $27(25.5)$ & $33(35.1)$ & $55(32.3)$ \\
\hline Renal failure & 1 & $157(44.1)$ & $41(38.7)$ & $38(40.4)$ & $78(50.0)$ \\
\hline NYHA II-IV & 55 & $237(78.7)$ & $59(68.6)$ & $67(80.7)$ & $111(84.1)$ \\
\hline NT-pro-BNP & 66 & 1409 [2112] & 1070 [1584] & $1071[1486]$ & 1751 [2466] \\
\hline
\end{tabular}

Values are mean (SD), median [IQR] or number (percentage);

Abbreviations: $\mathrm{BMI}=$ body mass index, $\mathrm{AF}=$ atrial fibrillation $($ on $\mathrm{EKG}), \mathrm{HF}=$ heart failure, $\mathrm{CAD}=$ coronary artery disease NYHA = New York Heart Association heart failure classification, NT-pro$\mathrm{BNP}=\mathrm{N}$-terminal pro-B-type natriuretic peptide.

The diagnosis renal failure is based on either chronic renal insufficiency or creatinine serum level greater than $100 \mu \mathrm{mol} / \mathrm{L}$ on admission. 
Table 2. Key echo measures (and measures of,) as both continuous variables and percent abnormal, along with the $\mathrm{N}$ for each measure (to indicate the extent of missing data for each measure) in the study sample overall and stratified by the number of echo abnormalities.

\begin{tabular}{|c|c|c|c|c|c|}
\hline \multirow[t]{2}{*}{ Characteristic } & \multirow{2}{*}{$\begin{array}{l}\text { Missing } \\
\mathrm{N}\end{array}$} & \multirow{2}{*}{$\begin{array}{l}\text { Overall } \\
\mathrm{N}=356\end{array}$} & \multicolumn{3}{|c|}{ Number of echo abnormalities } \\
\hline & & & $\begin{array}{c}<4 \\
N=106\end{array}$ & $\begin{array}{c}4 \\
N=94\end{array}$ & $\begin{array}{c}5 \text { to } 8 \\
\mathrm{~N}=156\end{array}$ \\
\hline LVEF, \% & 14 & $62.4(7.0)$ & $62.9(6.4)$ & $62.1(6.7)$ & $62.1(7.5)$ \\
\hline IVRT, ms & 20 & $91.8(31.2)$ & $93.8(20.7)$ & $93.2(30.3)$ & $89.5(37.3)$ \\
\hline$<55$ or $>110 \mathrm{~ms}, \%$ & & $118(33.1)$ & $18(17.0)$ & $27(28.7)$ & $73(46.8)$ \\
\hline DT, ms & 7 & $194(75)$ & $199(54)$ & $198(70)$ & $189(90)$ \\
\hline$<150$ or $>280 \mathrm{~ms}, \%$ & & $141(39.6)$ & $18(17.0)$ & $30(31.9)$ & $93(59.6)$ \\
\hline $\mathrm{E} / \mathrm{A}$ & 92 & $2.1(1.4)$ & $1.8(1.1)$ & $1.9(1.2)$ & $2.4(1.5)$ \\
\hline$<0.5$ or $>2, \%$ & & $168(47.2)$ & $32(30.2)$ & $40(42.5)$ & $96(61.5)$ \\
\hline $\mathrm{E}^{\prime}, \mathrm{cm} / \mathrm{s}$ & & $8.0(2.6)$ & $9.2(2.6)$ & $8.2(2.6)$ & $7.0(2.2)$ \\
\hline$<9 \mathrm{~cm} / \mathrm{s}, \%$ & & $227(63.8)$ & $44(41.5)$ & $56(59.6)$ & $127(81.4)$ \\
\hline $\mathrm{E} / \mathrm{E}^{\prime}$ & 24 & $12.8(6.1)$ & $9.1(3.5)$ & $11.8(5.8)$ & $16.0(6.1)$ \\
\hline$\geq 13, \%$ & & $138(38.8)$ & $7(6.60)$ & $27(28.7)$ & $104(66.7)$ \\
\hline LAVI, $\mathrm{mL} / \mathrm{m}^{2}$ & 18 & $47.6(16.1)$ & $40.2(14.3)$ & $46.6(15.8)$ & $52.8(15.4)$ \\
\hline$>34 \mathrm{~mL} / \mathrm{m}^{2}, \%$ & & $280(78.6)$ & $60(56.6)$ & $78(83.0)$ & $142(91.0)$ \\
\hline LVMI, g/m² VG6 & 104 & $125(37)$ & $106(36)$ & $127(32)$ & $137(35)$ \\
\hline LVH, \% & & 255 (71.6) & 43 (40.6) & $72(76.6)$ & $140(89.7)$ \\
\hline TR & 49 & $2.8(0.7)$ & $2.4(0.6)$ & $2.8(0.5)$ & $3.1(0.6)$ \\
\hline$\geq 2.8 \mathrm{~m} / \mathrm{s}$ & & $179(50.3)$ & $17(16.0)$ & 46 (48.9) & $116(74.4)$ \\
\hline
\end{tabular}

Values are mean (SD) or number (percentage); 
Abbreviations: $\hat{E}=$ mitral tissue Doppler É velocity, E/É = ratio of mitral Doppler E velocity to mitral tissue Doppler É velocity, DT = deceleration time, E/A = ratio of mitral E to A velocity, IVRT = isovolumetric relaxation time, $\mathrm{LAVI}=$ left atrial volume index, $\mathrm{LVEF}=$ left ventricle ejection fraction, $\mathrm{LVMI}=$ left ventricular mass index, $\mathrm{LVH}$ if $\mathrm{LVMI}>95 \mathrm{~g} / \mathrm{m}^{2}$ in women or $>115 \mathrm{~g} / \mathrm{m}^{2}$ in men, $\mathrm{TR}=$ tricuspid regurgitation 
Table 3. Rates (per 100 person-years) and hazard ratio (HR) for death from all causes or first hospitalization for heart failure according to diastolic parameters, with adjustment for age, gender and $\mathrm{EF}$ (first row) and further adjustment for BNP/pro-BNP quintiles (second row).

\begin{tabular}{|c|c|c|c|c|c|c|}
\hline Parameters & Value & $\mathrm{N}$ & No events & Event rates & HR (95\% CL) & $\mathrm{p}$-value \\
\hline \multirow[t]{7}{*}{ Number } & $<4$ & 106 & 56 & 25.5 & 1.00 & \\
\hline & 4 & 94 & 58 & 32.9 & $1.5(0.8-2.7)$ & 0.1594 \\
\hline & & & & & $1.4(0.7-2.6)$ & 0.3116 \\
\hline & 5 to 8 & 156 & 147 & 58.4 & $2.3(1.5-3.6)$ & 0.0002 \\
\hline & & & & & $2.0(1.3-3.3)$ & 0.0032 \\
\hline & 4 to 8 & 250 & 205 & 47.9 & $2.0(1.3-3.2)$ & 0.0020 \\
\hline & & & & & 1. $8(1.1-2.8)$ & 0.0189 \\
\hline \multirow{3}{*}{$\begin{array}{l}\text { Structural abnormality and } \\
\text { diastolic dysfunction }\end{array}$} & No & 81 & 39 & 21.2 & 1.00 & \\
\hline & Yes & 275 & 222 & 47.8 & $2.1(1.3-3.2)$ & 0.0020 \\
\hline & & & & & $1.7(1.0-2.7)$ & 0.0380 \\
\hline \multirow[t]{7}{*}{ Diastolic dysfunction } & 0 & 24 & 10 & 18.5 & 1.00 & \\
\hline & 1 & 107 & 59 & 27.9 & $1.5(0.6-4.2)$ & 0.4154 \\
\hline & & & & & $1.3(0.4-3.9)$ & 0.6875 \\
\hline & 2 & 97 & 70 & 37.3 & $2.1(0.8-5.8)$ & 0.1403 \\
\hline & & & & & $1.9(0.6-6.1)$ & 0.2505 \\
\hline & 3 & 124 & 120 & 64.4 & $3.0(1.1-8.0)$ & 0.0278 \\
\hline & & & & & $2.5(0.8-7.5)$ & 0.1146 \\
\hline
\end{tabular}

Diastolic dysfunction: grade $1=$ relaxation abnormality, grade 2 = pseudo-normalisation, grade $3=$ restrictivity. 


\section{Highlights}

- A model for grading the diastolic dysfunction of the heart is proposed.

- Most of the patients with suspected heart failure based on the Framingham criteria, increase of natriuretic peptides and normal ejection fraction met objective diagnostic echocardiographic criteria for heart failure with preserved ejection fraction according to the ESC guidelines.

- The ESC heart failure guidelines can be used for risk prediction in heart failure with preserved ejection fraction. 


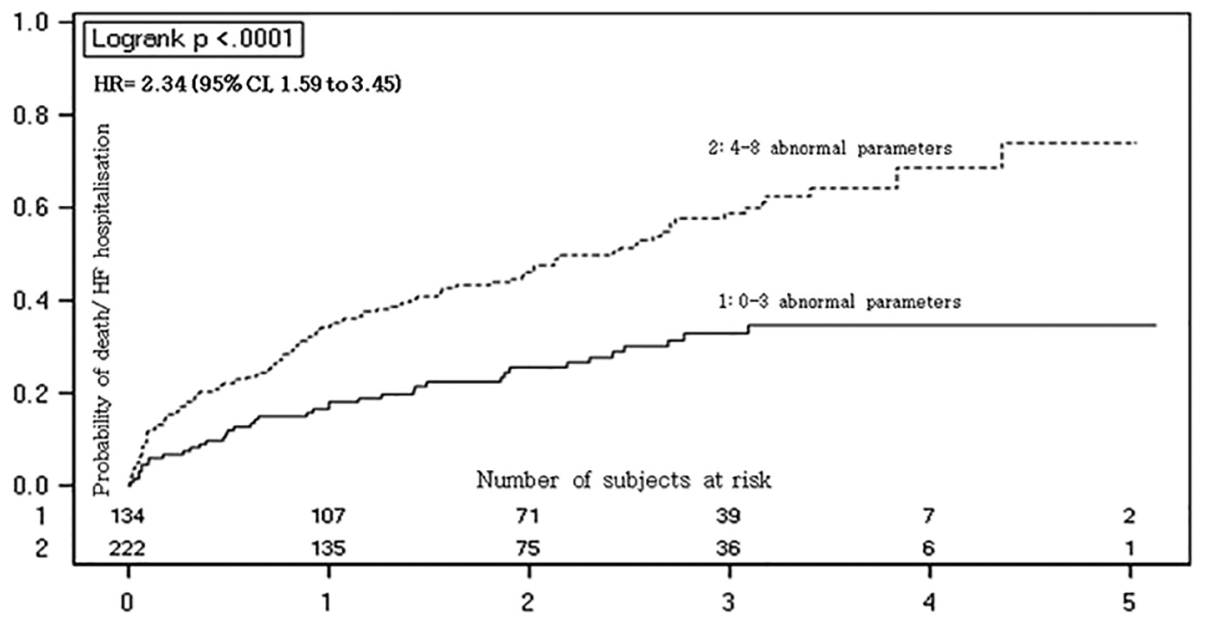

Follow-up in years

Figure 1 